\title{
Crystal engineering using functionalized adamantane
}

\author{
J. C. Garcia ${ }^{1}$, L. V. C. Assali ${ }^{1}$, W. V. M. Machado ${ }^{1}$, and J. F. Justo ${ }^{2}$ \\ 1 Instituto de Física, Universidade de São Paulo, \\ CP 66318, CEP 05315-970, São Paulo, SP, Brazil \\ 2 Escola Politécnica, Universidade de São Paulo, \\ CP 61548, CEP 05424-970, São Paulo, SP, Brazil
}

(Dated: June 25, 2010)

\begin{abstract}
We performed a first principles investigation on the structural, electronic, and optical properties of crystals made of chemically functionalized adamantane molecules. Several molecular building blocks, formed by boron and nitrogen substitutional functionalizations, were considered to build zincblende and wurtzite crystals, and the resulting structures presented large bulk moduli and cohesive energies, wide and direct bandgaps, and low dielectric constants (low- $\kappa$ materials). Those properties provide stability for such structures up to room temperature, superior to those of typical molecular crystals. This indicates a possible road map for crystal engineering using functionalized diamondoids, with potential applications ranging from space filling between conducting wires in nanodevices to nano-electro-mechanical systems.
\end{abstract}

PACS numbers: 


\section{INTRODUCTION}

The recent progresses in nanotechnology have allowed to envision building crystals with designed properties from bottom-up. This procedure, generally labeled as crystal engineering, could become a reality within the current available technologies if a number of conditions are properly observed $\stackrel{1}{1,2}$. The molecular building blocks (MBBs), the fundamental nanobricks, must carry strong intra-molecular bonding in order to guarantee internal molecular rigidity ${ }^{3}$. Moreover, the MBBs must contain appropriate chemical active centers to provide attractive and strong inter-molecular bonding, which ensures rigidity for the entire crystal. Although getting MBBs with those properties might be reasonably easy to achieve, the major challenge has been the synthesis, the process in which those MBBs are put together in organized and systematic fashion. There are essentially two ways to arrange them: physics- and chemistry-oriented methods. Physics methods, such as the atomic force microscopy, are used to manipulate single molecules onto surfaces, allowing positional control with sub-nanometric precision. However, those methods are fundamentally too slow because the molecules are manipulated individually and in sequence. On the other hand, chemistry methods, such as self-assembly, are more appealing to build nanocrystals in large scale.

The appropriate choice of MBBs is crucial in crystal engineering. Carbon-based nanostructures, such as nanotubes, buckballs, and polymers, have been considered as leading candidates to work as $\mathrm{MBBs}^{4}$. Diamondoids, also known as molecular diamond, have been recently included in this list of candidates ${ }^{3, \underline{5}} \underline{-10}$. Although diamondoids have been known for a long time, they have received great attention only after recent developments that allowed to manipulate and separate higher diamondoids $\frac{11}{}$.

Diamondoids face a major challenge to be used in crystal engineering, since molecular crystals made of pristine diamondoids have weak inter-molecular bonding and, consequently, are very brittle ${ }^{5}$. An additional challenge is to find a microscopic driving force to favor self-assembly. Chemical functionalization is a process in which those two points could be simultaneously addressed. For example, functionalized diamondoids could work as MBBs that can self-organize, using the inter-molecular interactions between chemically active sites in different functionalized molecules as the driving forces ${ }^{3}$. The resulting crystals could have strong inter-molecular bonds when compared to hydrogen-hydrogen interactions, that 
control inter-molecular bonding in typical molecular crystals.

In order to explore the concept of using functionalized diamondoids as MBBs, we used first principles calculations to devise crystals made of functionalized adamantane molecules with boron and/or nitrogen atoms. We found that the resulting crystals carried large cohesive energies and bulk moduli, which was a direct consequence of functionalization ${ }^{5}$. Additionally, those crystals present wide bandgaps and low dielectric constants, equivalent to properties of molecular crystals made of pristine adamantane ${ }^{5}$. All those properties allow to envision widespread applications, ranging from nano-electro-mechanical systems (NEMS) to insulating dielectrics in nanodevice interconnects or opto-electronic devices. This manuscript is organized as follows: section II presents the methodological aspects of the simulations, section III presents the structural, electronic and optical properties of the molecular crystals, discussing the role of functionalization. Finally, section $I V$ discusses the potential applications for those crystalline structures.

\section{METHODOLOGY}

The calculations were performed using the VASP package (Vienna ab initio simulation package) $)^{12}$ with periodic boundary conditions. The electronic exchange-correlation potential was described within the density functional theory in the generalized gradient approximation $\frac{13}{}$. The electronic wave-functions were described by a projector augmented wave (PAW) method ${ }^{14}$, taking a plane-wave basis set with an energy cutoff of $450 \mathrm{eV}$. Convergence in total energy was set to $0.1 \mathrm{meV} /$ atom between two self-consistent iterations. For a crystal in a certain lattice parameter, configurational optimization was performed by considering relaxation in all atoms, without any symmetry constrain, until forces were lower

than $3 \mathrm{meV} / \AA$ in any atom. The Brillouin zone was sampled by a Monkhorst-Pack grid of about $100 \mathrm{k}$-points $\frac{15}{5}$. The complex dielectric tensors were computed within the framework of the random phase approximation ${ }^{16}$. The dielectric constant $(\kappa)$ was computed as the photon zero-energy limit of the real part of the dielectric tensor.

The reliability of our results using this solid-state-based methodology was checked by comparing the properties of isolated adamantane molecules, in pristine and functionalized forms, with the ones using a chemistry-based methodology ${ }^{17}$ ? . In terms of structural properties of all molecules, both methodologies provide results for interatomic distances (differences 
lower than $0.01 \AA$ ) and angles (differences lower than $0.1^{0}$ ) in excellent agreement with each other.

\section{RESULTS}

Adamantane, the smallest diamondoid, has a $\mathrm{C}_{10} \mathrm{H}_{16}$ chemical composition, which comprises of one diamond-like carbon cage with hydrogen atoms passivating the remaining bonds. It contains two types of carbon atoms: four $\mathrm{C}(1)$ and $\operatorname{six} \mathrm{C}(2)$ atoms. $\mathrm{A} \mathrm{C}(1)$ is bound to three $\mathrm{C}(2)$ 's and one $\mathrm{H}$ atom, while a $\mathrm{C}(2)$ is bound to two $\mathrm{C}(1)$ 's and two H's. Figure 1a presents the structure and schematic representation of an adamantane molecule. Functionalization in the $\mathrm{C}(1)$ or $\mathrm{C}(2)$ sites provides respectively four or six chemically active sites to allow inter-molecular bonding. In principle, a larger number of functionalized sites, such as the $\mathrm{C}(2)$ ones, would be desirable, since it could lead to a larger number of inter-molecular bonds, and consequently stiffer nanostructures ${ }^{3}$. However, there are strong evidences that functionalization with boron or nitrogen atoms is favorable in the $\mathrm{C}(1) \operatorname{sites}^{18}$.

Chemical functionalization, by replacing the $\mathrm{C}(1)-\mathrm{H}$ groups with nitrogen and/or boron atoms, leads to interesting molecules to be used as MBBs: tetra-aza-adamantane $\left(\mathrm{N}_{4} \mathrm{C}_{6} \mathrm{H}_{12}\right)$, tetra-bora-adamantane $\left(\mathrm{B}_{4} \mathrm{C}_{6} \mathrm{H}_{12}\right)$, and di-aza-di-bora-adamantane $\left(\mathrm{B}_{2} \mathrm{~N}_{2} \mathrm{C}_{6} \mathrm{H}_{12}\right)$, which are labeled here TA, TB and DADB, respectively, and are schematically represented in figure 1b. Those molecules are very stable, providing four chemically active sites, still preserving the strong intra-molecular bonding of the original adamantane molecule ${ }^{6}$. Considering those molecules as MBBs, self-assembly could take place by using, as the driving force, the dipolar interaction between the boron site in one molecule and the nitrogen site in the neighboring one.

In order to explore the potentialities of crystal engineering using functionalized adamantane, we devised a number of molecular crystals that could be grown by self-assembly. We should emphasize that although we are here using the term molecular crystal, the intermolecular interactions in those crystals are controlled by covalent bonding $\underline{6}$, instead of the weak dispersive attractive interactions usually observed in typical molecular crystals. The functionalized adamantane molecules, with four chemically active sites, have a tetrahedral symmetry, which favors crystalline organization in zincblende (ZB) or wurtzite (WZ) struc-

tures. The tetrahedral symmetry of such arrangement is indicated in figure 1r, evidencing 
that inter-molecular bonding is controlled by the B-N interactions. For example, the crystal formed by a basis of TA and TB molecules in a ZB configuration, labeled here as ZB-TA+TB, a TA molecule sits in the lattice origin $(0,0,0)$ and a TB one sits in the $(1 / 4,1 / 4,1 / 4) a$ position, where $a$ is the crystalline lattice parameter. This crystal could be equivalently formed by a basis consisting of two DADB molecules, appropriately arranged to provide only B-N inter-molecular bonds. In this last case, due to the equivalence of the two molecules in the basis, we labeled as diamond-2DADB.

Crystalline structures with the same molecules discussed in the previous paragraph, but arranged in a WZ configuration, are labeled WZ-TA+TB and WZ-2DADB. However, the WZ structure contains four molecules in the crystalline basis, in contrast to only two in ZB structure. This WZ structure could be described by a hexagonal structure plus a basis of four molecules. For example, the WZ-TA+TB is formed by two TA molecules sitting in $(0,0,0)$ and $(a / 3,2 a / 3, c / 2)$ positions and two TB molecules sitting in $(0,0, u c)$ and $(a / 3,2 a / 3, u c+c / 2)$ positions, where $a$ and $c$ are lattice parameters and $u$ is an internal parameter.

Although the TA+TB and 2DADB crystals, in both crystalline configurations, are isomeric, they carry important differences in terms of self-assembly. The TA+TB crystals could be grown layer by layer, with sub-nanometric precision, by introducing the crystal seed alternately in solutions rich in tetra-aza- and tetra-bora-adamantane molecules. In this case, the driving force for self-assembly comes from attractive Coulomb interactions between the different molecules in solution. An equivalent procedure to build 2DADB crystals would be considerably more difficult using solutions rich in di-aza-di-bora-adamantane molecules, since all molecules are in the same charge state, and the driving force should be controlled by the weak attractive multipole interactions.

The stability of the molecular crystals can be quantified by the cohesive (or lattice) energy, the energy gain between a configuration in which the molecules are infinitely separated and one in a crystalline phase. Figure 2 presents the total energy variation as a function of the crystalline volume for the ZB- and WZ-2DADB. As discussed in the previous paragraphs, the molecular crystal in a ZB configuration contains two functionalized molecules (one TA plus one TB molecule) while the WZ one contains four molecules (two TA plus two TB molecules), and therefore the total energy is presented per pair of functionalized molecules, in order to allow a proper comparison of energies between the ZB and WZ crystals. The results indicated strong cohesion for both crystalline structures, although with a small favoring for 
the ZB configuration over the WZ one. Table 1 summarizes the properties of crystals with different compositions, indicating favoring of the ZB structure for all those crystals.

It has been recently shown that cohesion, in molecular crystals made of functionalized adamantane with boron and nitrogen atoms, is essentially controlled by the strong intermolecular B-N bonding, and intra-molecular bonds are only slightly affected by the presence of the neighboring functionalized molecules ${ }^{6}$. Therefore, cohesive energy should be essentially associated with the binding energy of the inter-molecular B-N bonds. For the diamond-2DADB crystal, cohesive energy per inter-molecular $\mathrm{B}-\mathrm{N}$ bond is $1.85 \mathrm{eV}$, which is considerably smaller than the one for a B-N bond in hexagonal boron nitride solids of $\approx 6.9$ $\mathrm{eV}$, computed using the same theoretical framework. However, this value is large enough to guarantee stability for the molecular crystal. Additionally, those crystals present large bulk moduli, in the 20-42 GPa range, when compared to values of around 10 GPa for typical organic molecular crystals 19 . The 2DADB crystals provide larger cohesive energies and bulk moduli than the TA+TB ones. The stiffer 2DADB crystals resulted from the smaller B-N inter-molecular bonds (1.725-1.752 $\AA$ ) when compared to the respective ones (1.907$1.983 \AA$ ) in the TA+TB crystals. The inter-molecular B-N bonds have a dative covalent character in all crystals, such that the B-N bonds are essentially formed by the electrons coming from the non-bonding 2 p orbitals (lone pair) in nitrogen functionalized adamantane. This covalent behavior was already observed in the interaction between two functionalized diamantane molecules (one with nitrogen and the other with boron), as discussed in Ref. This strong inter-molecular bonding contrasts with bonding in typical molecular crystals, such as crystals made of pristine adamantane $e^{5}$, in which bonding is controlled by the weak dispersive attractive potentials.

We also explored the possibility of building molecular crystals using the radical tetraadamantyl (RTA) as a MBB. This molecule is formed by removing the four hydrogen atoms bonded to $\mathrm{C}(1)$ atoms in adamantane (Fig. 1b). Although this is only a hypothetical molecular configuration, our calculations indicated that it is mechanically stable and maintains the cage-like structure of the original adamantane molecule. Table \presents the properties of crystals made of tetra-adamantyl molecules, called ZB- or WZ-2RTA, indicating large cohesive energies and bulk moduli. The inter-molecular C-C bonds (1.608-1.632 $\AA$ ) in both configurations are only a little longer than the respective ones in diamond $(1.54 \AA)$. The binding energy per inter-molecular bond is around $6 \mathrm{eV}$, which is very close to the cohesive 
energy per $\mathrm{C}-\mathrm{C}$ bond in diamond $(7.5 \mathrm{eV})$. This indicates that the inter-molecular bonds diamond-2RTA and WZ-2RTA are essentially covalent and equivalent to bonds in diamond.

Our results have so far shown that crystals, made of functionalized adamantane, have appropriate mechanical properties for several applications in NEMS. In order to evaluate other potentialities, we explored their electronic and dielectric properties. All molecular crystals investigated here have wide and direct bandgaps (between 3.8 and $4.2 \mathrm{eV}$ ), as shown in table I. These results suggest potential applications for opto-electronic devices in the ultraviolet region. Those values are about $1 \mathrm{eV}$ lower than the bandgaps computed for molecular crystals made of pristine adamantane $(5.1 \mathrm{eV})$ using the same methodology. These results are fully consistent with recent experimental data, which found an $1 \mathrm{eV}$ reduction in the principal gap of adamantane as result of nitrogen functionalization (to form tetraaza-adamantane molecules) $)^{21}$. As it has been recently pointed out, the nitrogen atoms incorporated in the $\mathrm{C}(1)$ sites of adamantane introduce $2 \mathrm{p}$ nitrogen-related energy levels in the top of valence band of adamantane, reducing the principal gap by $1.3 \mathrm{eV}^{\underline{6}}$.

Figure 3 shows the electronic band structure of the diamond-2DADB and diamond-2RTA crystals. The band structure of the ZB-TA+TB crystal ${ }^{6}$ is similar to that of the diamond2DADB one, in terms of dispersion, effective masses and gaps. In the diamond-2DADB crystal, the top of the valence band presents a small dispersion, while the bottom of the conduction band presents a considerably larger dispersion, which is totally similar to the band structure of pure adamantane crystal ${ }^{5}$. On the other hand, the diamond-2RTA crystal presents large dispersion in both band limits, evidencing the strong covalent bonding controlling inter-molecular interactions. The effective masses for electrons in the conduction band and holes in the valence bands are presented in table I. The results indicate that effective masses for holes $\left(m_{h}^{*}\right)$ are larger than masses for electrons $\left(m_{e}^{*}\right)$ for all crystals, indicating favoring for electron transport over hole one.

Figure 4 shows the real and imaginary dielectric functions $(\varepsilon)$ for the diamond-2DADB molecular crystal as a function of the photon energy. Those functions are similar in all the other molecular crystals investigated here (TA+TB and 2RTA). The dielectric constant $(\kappa)$, computed as the photon zero-energy limit of $\operatorname{Re}(\varepsilon)$, is in the 2.8-3.0 range for all molecular crystals, which is in the same range of that constant for a molecular crystal made of pristine adamantane (2.7) in a tetragonal configuration, computed using the same methodology. Those values are fully consistent with other calculations on molecular crystals made of 
pristine diamondoids (in the $2.46-2.68$ range ${ }^{20}$. Therefore, these molecular crystals could be classified as low- $\kappa$ materials, in the context of electronic circuits that consider the dielectric constant of silicon oxide $(\kappa=3.9)$ as a reference value. These results suggest potential applications as dielectric materials to work as space filling between nanodevice interconnects to minimize parasitic capacitances.

\section{SUMMARY}

In summary, we have shown that adamantane molecules, either chemically functionalized or in the radical form, are potential candidates to work as molecular building blocks for nanostructure self-assembly. The crystals formed by those molecules, in zincblende and wurtzite configurations, presented favorable mechanical properties, such as large bulk moduli as large as $70 \mathrm{GPa}$, which make them very stable at room temperatures or even higher. All those molecular crystals presented wide and direct electronic bandgaps in the 3.8 to $4.2 \mathrm{eV}$ range, indicating potential applications in opto-electronics in the ultraviolet region. However, those theoretical gap values should be considered as lower limits, since they are generally underestimated, when compared to experimental data, by at least $1 \mathrm{eV}$ using the theoretical framework of the density functional theory $\underline{\underline{6}} \underline{\underline{8}}$.

Those molecular crystals could be used as low- $\kappa$ dielectrics between interconnects in nanodevices, with dielectric constants in the 2.8-3.0 range. In the search for low- $\kappa$ dielectrics, scientists have already found materials in which that constant could be as low as 2.0, such as porous silicon and silicon oxide materials. Porous materials tend to be very brittle, and therefore unsuitable for large scale integration in device technologies. On the other hand, the molecular crystals presented several advantages over those low- $\kappa$ dielectric materials, since they provide simultaneously large bulk moduli, which guarantees mechanical stability.

\section{Acknowledgments}

This work was partially supported by the Brazilian Agencies CNPq and CAPES. The calculations were performed at the computational facilities of CENAPAD-São Paulo.

1 Wang X, Zhuang J, Peng Q and Li Y D 2005 Nature 437121 
2 Sharma C V K 2002 Crystal Growth Design 2465

3 Merkle R C 2000 Nanotechnology 1189

4 Moulton B and Zaworotko M J 2001 Chem. Rev. 1011629

5 Sasagawa T and Shen Z-X 2008 J. Appl. Phys. 104073704

6 Garcia J C, Justo J F, Machado W V M and Assali L V C 2009 Phys. Rev. B 80125421

7 Garcia J C, Justo J F, Machado W V M and Assali L V C 2010 Diamond Relat. Mater. 19837

8 McIntosh G C, Yoon M, Berber S and Tománek D 2004 Phys. Rev. B 70045401

9 Drummond N D 2007 Nature Nanotechnology 2462

10 Marsusi F and Mirabbaszadeh K 2009 J. Phys.- Condens. Mat. 21215303

11 Dahl J E, Liu S G and Carlson R M K 2003 Science 29996

12 Kresse G and Furthmüller J 1996 Phys. Rev. B 5411169

13 Perdew J P, Burke K and Ernzerhof M 1996 Phys. Rev. Lett. 773865

14 Kresse G and Joubert D 1999 Phys. Rev. B 591758

15 Monkhorst H J and Pack J D 1976 Phys. Rev. B 135188

16 Gajdos M, Hummer K, Kresse G, Furthmüller J and Bechstedt F 2006 Phys. Rev. B 73045112

17 Frisch M J, Trucks G W, Schlegel H B et al., GAUSSIAN 98, Revision A.11.2 (Gaussian, Inc., Pittsburgh, PA, 2001).

18 Kampermann S P, Sabine T M, Craven B M and McMullan R K 1995 Acta Cryst. A 51489

19 Day G M, Price S L and Leslie M 2001 Crystal Growth Design 113

20 Clay W A, Sasagawa T, Kelly M, Dahl J E, Carlson R M K, Melosh N and Shen Z-X 2008 Appl. Phys. Lett. 93172901

21 Landt L, Kielich W, Wolter D, Staiger M, Ehresmann A, Möller T and Bostedt C 2009 Phys. Rev. B 80205323 
TABLE I: Properties of molecular crystals in zinc-blende (ZB), wurtzite (WZ), and diamond configurations, using several building blocks: tetra-aza-adamantane plus tetra-bora-adamantane molecules (TA+TB), two di-aza-di-bora-adamantane molecules (2DADB), and two radical tetraadamantyl molecules (2RTA). The table presents the interatomic distance of the inter-molecular bond $(d)$, lattice $(a, c)$ and internal $(u)$ parameters, cohesive energy per pair of molecules $\left(\mathrm{E}_{c}\right)$, bulk modulus (B) and its pressure derivative (B'), electronic bandgap $\left(\epsilon_{g}\right)$, valence band width (VBW), dielectric constant $(\kappa)$, and hole $\left(m_{h}^{*}\right)$ and electron $\left(m_{e}^{*}\right)$ effective masses, given in terms of the free electron mass $\left(m_{0}\right)$.

\begin{tabular}{|c|c|c|c|c|c|c|}
\hline & $\mathrm{ZB}-\mathrm{TA}+\mathrm{TB}$ & $\mathrm{WZ}-\mathrm{TA}+\mathrm{TB}$ & diamond-2DADB & WZ-2DADB & diamond-2RTA & WZ-2RTA \\
\hline$d(\AA)$ & 1.907 & 1.983 & 1.725 & 1.752 & 1.608 & 1.632 \\
\hline$a(\AA)$ & 11.45 & 8.13 & 11.29 & 8.02 & 11.00 & 7.81 \\
\hline$c(\AA)$ & - & 13.32 & - & 13.11 & - & 12.73 \\
\hline$u$ & - & 0.377 & - & 0.378 & - & 0.382 \\
\hline $\mathrm{E}_{c}(\mathrm{eV})$ & 1.81 & 1.67 & 3.70 & 3.32 & 12.11 & 11.76 \\
\hline $\mathrm{B}(\mathrm{GPa})$ & 20 & 16 & 43 & 42 & 69 & 72 \\
\hline $\mathrm{B}^{\prime}$ & 10.9 & 10.8 & 7.3 & 7.1 & 5.1 & 5.4 \\
\hline$\epsilon_{g}(\mathrm{eV})$ & 3.9 & 3.8 & 4.1 & 4.2 & 4.4 & 4.2 \\
\hline $\mathrm{VBW}(\mathrm{eV})$ & 21.3 & 21.3 & 18.9 & 19.1 & 16.6 & 16.7 \\
\hline$\kappa$ & 2.9 & 2.8 & 2.9 & 2.8 & 3.0 & 2.9 \\
\hline $\mathrm{m}_{\mathrm{e}}^{*} / \mathrm{m}_{0}$ & 0.49 & 0.53 & 0.52 & 0.57 & 0.51 & 0.56 \\
\hline $\mathrm{m}_{\mathrm{h}}^{*} / \mathrm{m}_{0}$ & 2.7 & 3.2 & 2.7 & 3.4 & 1.3 & 1.2 \\
\hline
\end{tabular}


(a)
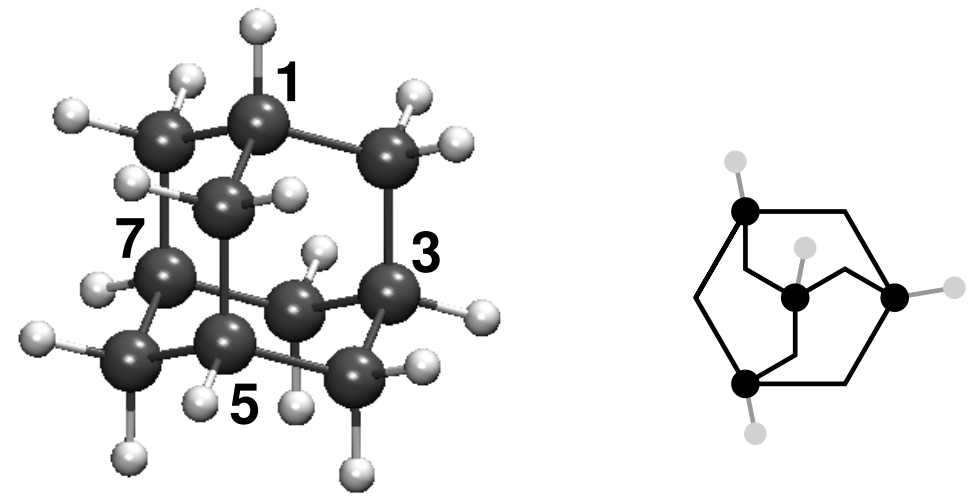

(b)
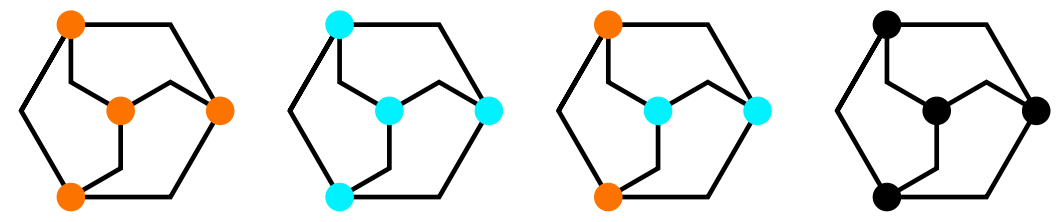

(c)

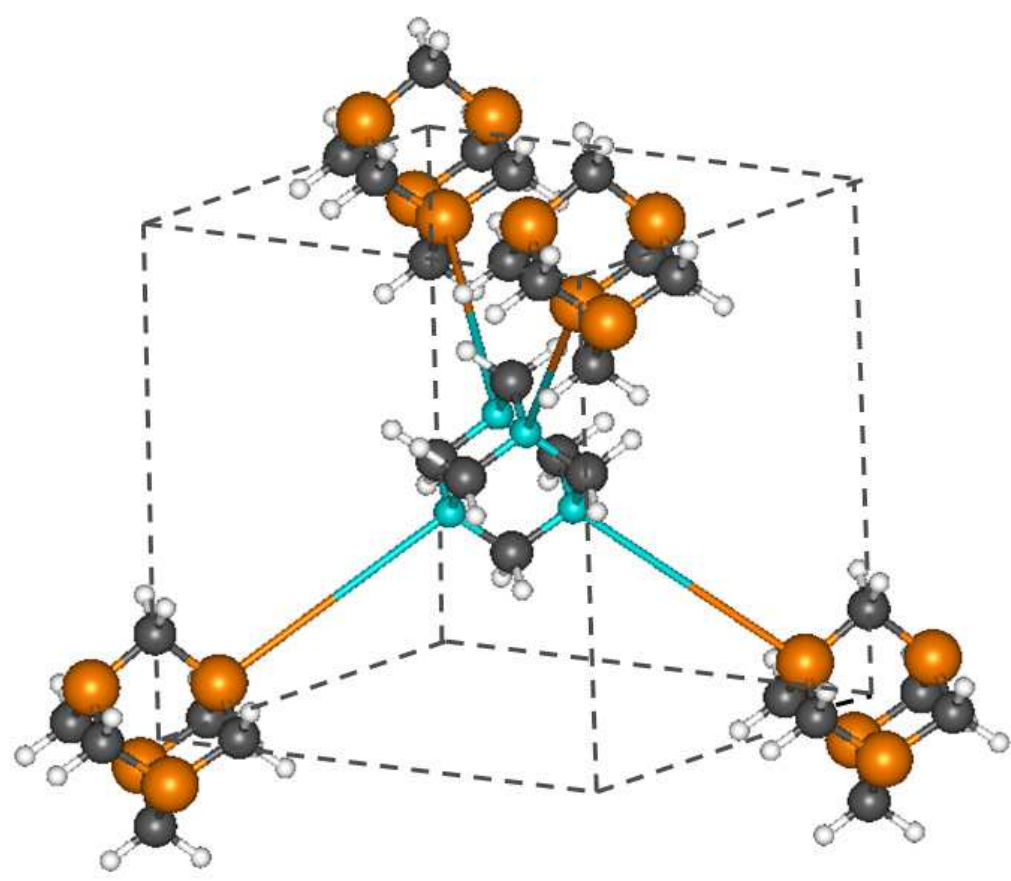

FIG. 1: (Color online) (a) Atomic configuration and schematic representation of pure adamantane molecule. The $\mathrm{C}(1)$ atoms are labeled by the numbers $1,3,5$, and 7 . The schematic representation shows only the $\mathrm{C}(1)$ and their respective hydrogen atoms. (b) Functionalized molecules: tetraaza-adamantane (1,3,5,7-aza-adamantane), tetra-bora-adamantane (1,3,5,7-bora-adamantane), diaza-di-bora-adamantane (1,3-aza-5,7-bora-adamantane), and tetra-adamantyl (1,3,5,7-adamantyl). (c) Configuration of a tetra-aza-adamantane molecule in a tetrahedral environment of four tetrabora-adamantane neighbors. Black, blue, orange, and gray circles represent respectively carbon, nitrogen, boron, and hydrogen atoms. 


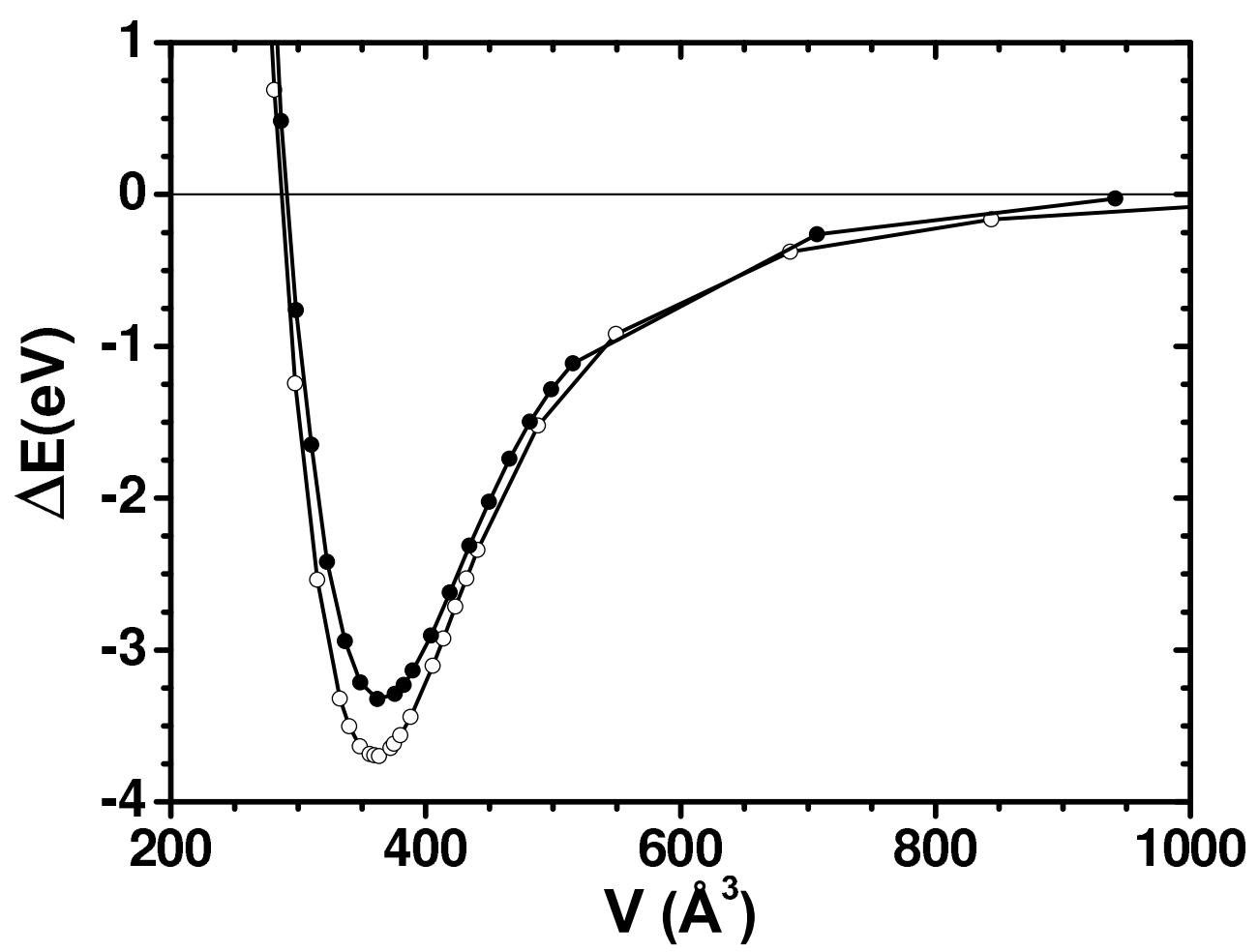

FIG. 2: Total energy variation per pair of molecules $(\Delta \mathrm{E})$, as a function of their crystalline volumes (V), for the diamond-2DADB (open symbols) and WZ-2DADB (black symbols) crystals. The reference energy is the one for infinitely separated molecules. 


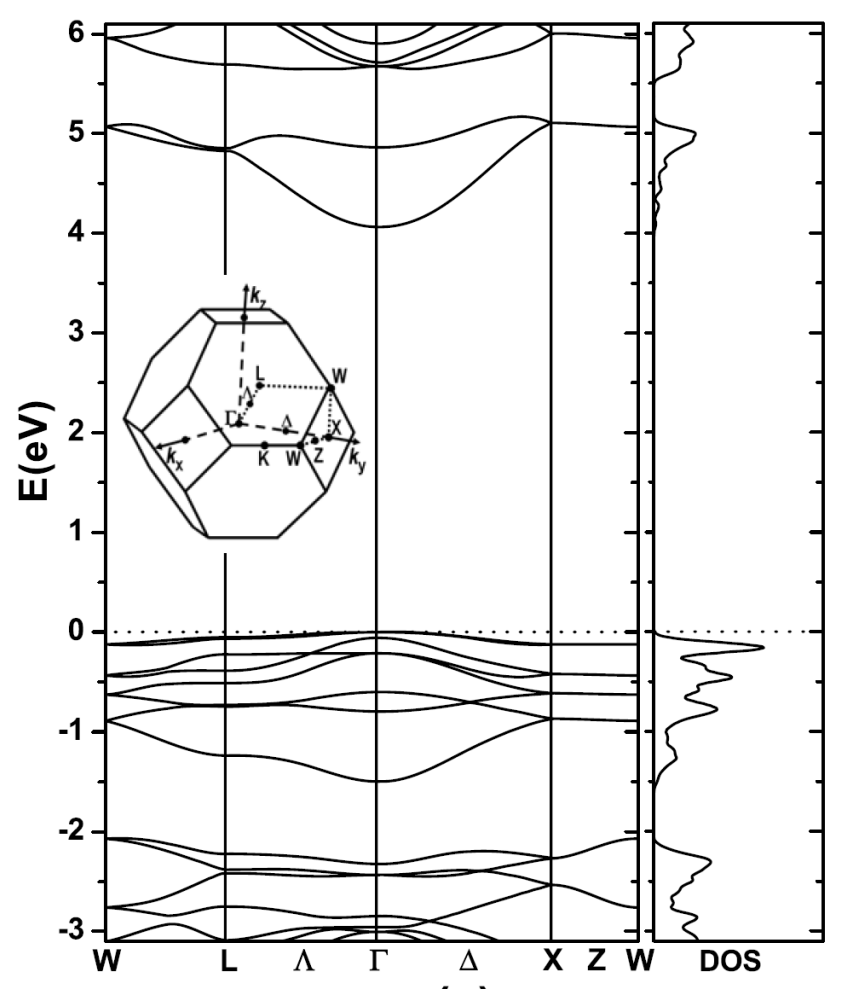

(a)

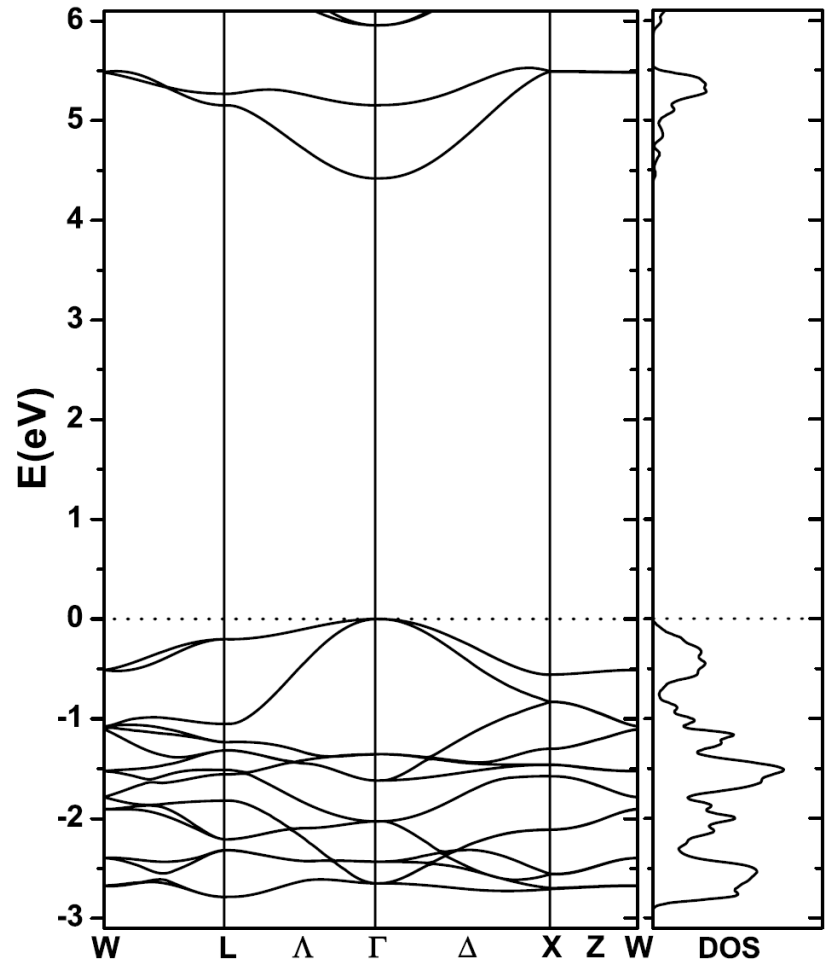

(b)

FIG. 3: Band structure, around the gap region, along several high symmetry directions and density of states (DOS) of the (a) diamond-2DADB and (b) diamond-2RTA crystals. The inset in (a) shows the first Brillouin zone and the respective high symmetry points. The bandgap is defined as the difference in energy between highest occupied (dashed lines) and lowest unoccupied states. 


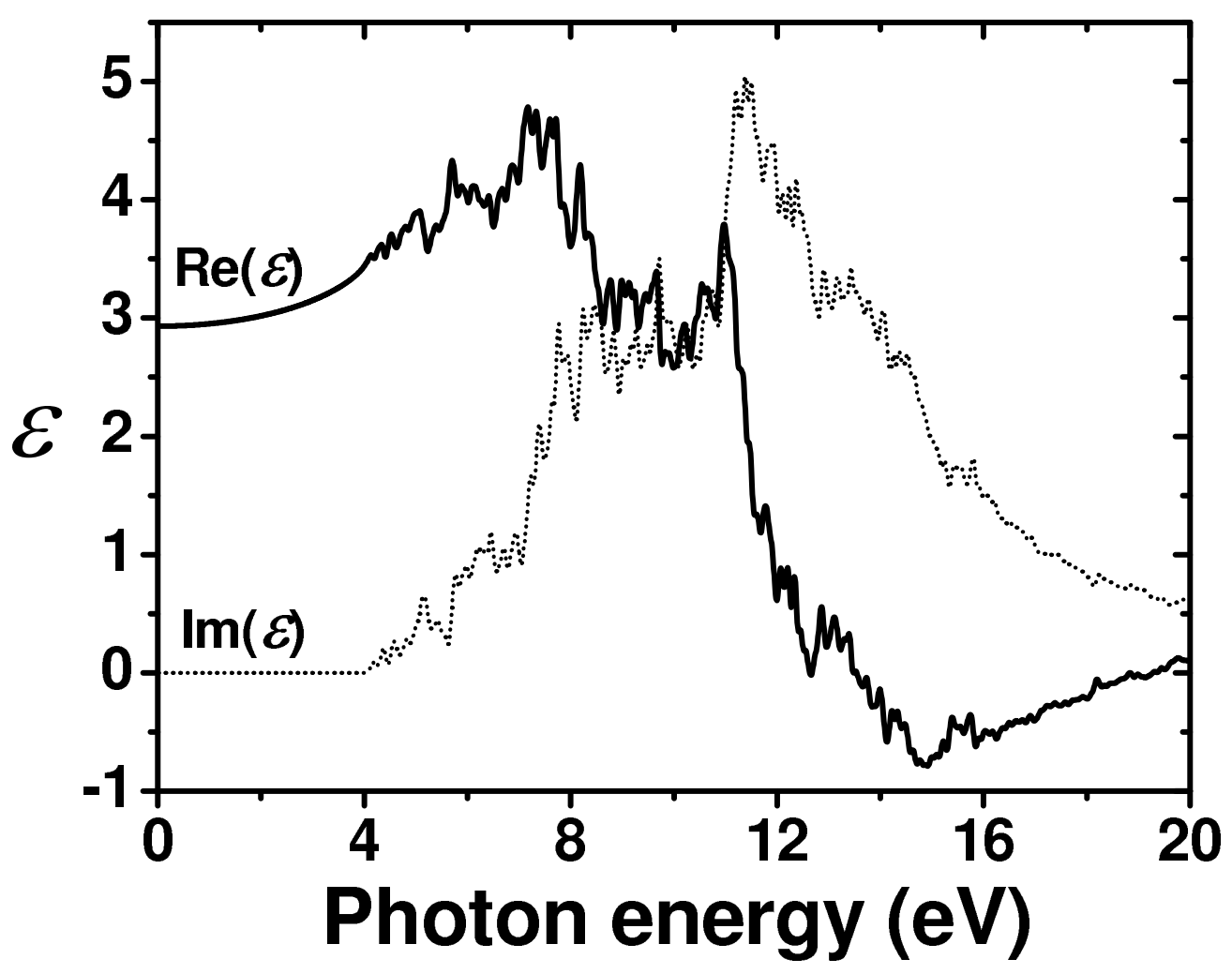

FIG. 4: Real and imaginary parts of the dielectric function $(\varepsilon)$, versus the photon energy, for the diamond-2DADB crystal. The dielectric constant is the limit of zero-photon energy of $\operatorname{Re}(\varepsilon)$. 